\title{
Inverted duplications are recurrent rearrangements always associated with a distal deletion: description of a new case involving $2 q$
}

\author{
Maria Clara Bonaglia ${ }^{1}$, Roberto Giorda ${ }^{1}$, Gerardina Poggi ${ }^{1}$, Maria Elisabetta Raggi ${ }^{1}$, \\ Elena Rossi ${ }^{3}$, Anna Baroncini ${ }^{2}$, Sabrina Giglio ${ }^{3}$, Renato Borgatti ${ }^{1}$ and Orsetta Zuffardi ${ }^{3,4}$
}

\author{
${ }^{1}$ IRCCS E. Medea, Bosisio Parini, Lecco; ${ }^{2}$ Servizio Genetica Medica Azienda USL, Imola; ${ }^{3}$ Biologia Generale e \\ Genetica Medica, Università Pavia; ${ }^{4}$ Laboratorio Citogenetica, Ospedale San Raffaele, Milan, Italy
}

\begin{abstract}
We studied the case of a subject with an inverted duplication of $40 \mathrm{cM}$ of 2 q33-q37 concurrent with a $10 \mathrm{cM}$ deletion of the distal $2 q$, the latter not being detectable by cytogenetics. Microsatellite analysis demonstrated the absence of maternal alleles in the deleted region and a double dosage for one of the maternal alleles in the duplication region. We hypothesised that this type of rearrangement occurs at meiosis I, while the two homologues are synapsed for most of their length. The presence of inverted duplicons in the same chromosome arm would favour the partial refolding of one homologue into itself so leading to the intrachromatid synapsis and recombination of the inverted repeats. The arising recombinant chromosome is deleted for the region beyond the most distal repeat and with the chromatids joined together at the level of the region located between the two duplicons. At meiosis II, the two linked chromatids can join the opposite poles provided that a breakage between the two centromeres occurs leading to a duplicated/deleted chromosome and a simply deleted chromosome. This model can be extended to all the so-called inverted duplication cases and to part of the terminal deletions. In fact the finding that, in our invdup(2q), the entire $40 \mathrm{cM}$ duplication region involves only one of the two maternal alleles, indeed indicates that the abnormal crossover occurs between sister chromatids. The phenotype associated with our $2 q$ rearrangement led us to narrow the critical region for the Albright-like syndrome to $10 \mathrm{cM}$ in the subterminal 2q region. European Journal of Human Genetics (2000) 8, 597-603.
\end{abstract}

Keywords: chromosome 2q; inverted duplications; deletion; inverted duplicons; Albright-like syndrome

\section{Introduction}

Chromosome inverted duplications were described as a new type of chromosome rearrangement ('mirror duplications') in 1977. ${ }^{1}$ At that time, a similar rearrangement had already been interpreted as an inverted duplication/deficiency ${ }^{2}$ but the molecular demonstration that the two imbalances, duplication and deletion, were concomitant, was made only 11 years later on a case of invdup(8p). ${ }^{3}$ The association inverted duplication/deletion had then been extended to all

Correspondence: Orsetta Zuffardi, Biologia Generale e Genetica Medica, Via Forlanini 14, 27100 Pavia, Italy. Tel: +390382 507733;

Fax: +390382 525030; E-mail: zuffardi@unipv.it

Received 22 December 1999; revised 31 March 2000; accepted 4 April 2000 invdup(8p)s $s^{4}$ and has been reported for several other chromosomes, its size being limited to the viability of the imbalance itself. Examples are those concerning $1 q^{5} 3 p,{ }^{6}$ $5 p,{ }^{7} 7 q,{ }^{8,9} 9 p,{ }^{10} 10 p$ and $10 q,{ }^{8}, 21 q,{ }^{11}$ and $X p .^{12}$ Although several authors hypothesised mechanisms that could account for the origin of such duplication/deficiencies, ${ }^{2,4,6,8}$ the causes of such rearrangements are still unclear.

We studied the case of a malformed child with an inverted duplication of $2 q$ spanning no less than $40 \mathrm{cM}$ in which we demonstrated a concomitant deletion for the distal $10 \mathrm{cM}$ that was not suspected on cytogenetic grounds. Studies of DNA polymorphisms led us to refine our previous hypothesis on the origin of inverted duplicated/deleted chromosomes ${ }^{4}$ and to explain the origin of some pure terminal deletions. According to the mechanism we propose, each of these 
rearrangements is mediated by inverted duplicons which favour intrachromatid misalignment and recombination.

\section{Clinical report}

The proband is a 7-year-old female, the only child of healthy unrelated parents. The son of a paternal cousin is affected by a form of spinocerebellar ataxia (not otherwise specified). She was born after an uneventful pregnancy after 42 weeks' gestation, with an Apgar score of 8 at $1 \mathrm{~min}$ and 10 at $5 \mathrm{~min}$. She was delivered by Caesarean section because of failure to progress in labour. Birthweight was $3.9 \mathrm{~kg}$ (90th percentile), birth length $54 \mathrm{~cm}$ (97th percentile) and head circumference $36 \mathrm{~cm}$ (75th percentile). Her parents were in the 50th centile for height and weight. At birth she showed prominent eyes, anti-mongoloid slanting of the palpebral fissures, hypertelorism, depressed nasal bridge, anteverted nostrils, small mouth with thin upper lip, short neck, low-set and small ears, small mandible, chest circumference $38 \mathrm{~cm}$ ( $>97$ th percentile) and widely spaced nipples, arachnodactyly of hands and feet with bilateral clinodactyly of the fifth finger, and external genital hypertrophy.

Routine biochemical investigations, blood and urine amino acids, fundus oculi and abdominal ultrasonography were normal. Echocardiography showed patent ductus arteriosus, pulmonary artery dilatation with mild left ventricle hypertrophy without ECG abnormalities. Chest and skull $\mathrm{X}$-rays were normal. MRI showed mild cortical atrophy with enlarged lateral ventricles, agenesis of the cerebellar vermis and severe hypoplasia of both cerebellar hemispheres. All developmental milestones were delayed: she walked at 20 months, she uttered her first words at 3 years of age. Rehabilitation (physiokinetic therapy or speech therapy) was performed until 6 years of age. Myopia and an alternating divergent squint were observed at 3years of age without funduscopic alterations. At the same age she suffered from epilepsy (generalised atonic seizures and/or drops attacks) which could be well controlled with valproic acid therapy. At present, the child is seizure-free and has not taken antiepileptic drugs for two years. Currently, at 7 years of age, her height is $129 \mathrm{~cm}$ (90th-97th percentile), weight $30 \mathrm{~kg}$ (90th-97th percentile), and occipito-frontal circumference (OFC) $52 \mathrm{~cm}$ (50th-75th percentile); facial dysmorphism is still present, skeletal X-rays show dorso lumbar scoliosis with concavity to the left. Neurological investigation reveals general ised muscular hypotrophy, minor cerebellar signs and ataxic ambulation. Moderate mental retardation (IQ $=49$ ) and severe speech impairment are present. Behavioural problems are also present: at 3 years of age she was hyperkinetic and aggressive; subsequently, she showed an oppositional and provocative attitude, both with adults and peers. At present, these behavioural patterns are less marked, even if when frustrated, she occasionally experiences self-mutilation episodes (she tears her hair).

\section{Materials and methods Cytogenetics investigations}

Chromosome analysis was performed on proposita and parents' blood using standard high-resolution techniques. ${ }^{13}$ Whole chromosome painting with a chromosome2-specific library (Vysis) was done on metaphase chromosome preparations.

Fluorescent in situ hybridisation (FISH) to chromosome preparations from the patient was performed as described ${ }^{14}$ with YACs containing chromosome 2 specific sequences from several locations on the $q$ arm, selected according to Genome Database (www.genome.wi.mit.edu/cgi-bin/contig/phys- map). YACs DNA was labelled with biotin and/or digoxigenin using nick translation. Three-colour FISH was performed with YACs 944E6 (D2S339; 2q33), 931C8 (D2S396: 2q34) and 854D9 (WI-6310: 2q37.3) labelled with biotin, dioxigenin and a one-to-one mix of biotin and digoxigenin, respectively. The labelled probes were visualised with FITCavidin (Vector) or rhodaminelabelled antidigoxigenin (Boehringer) and the chromosomes were counterstained with DAPI (Sigma). Hybridisations were analysed with a Zeiss Axioplan epifluorescence microscope and images were captured with the Power Gene FISH System (PSI).

\section{DNA analyses}

DNA was extracted from $3 \mathrm{ml}$ of peripheral blood with standard protocols. Polymorphic loci were selected based on their position on the Généthon genetic linkage map (〈www.genome.wi.mit.edu/cgi-bin/contig/phys-map〉) and the Genetic Location Database (LDB) map (/cedar.genetics.soton.ac.uk/public - html//). Locus information and primer sequences are available from the Genome Database (GDB) ( $\langle g d b w w w . g d b . o r g /\rangle)$. For all amplimers unlabelled reverse primers were purchased from Life Technologies, forward primers, labelled with 5-FAM, Hex or Tet dyes were obtained from MWG Biotech. PCRs were carried out using standard protocols (Perkin-Elmer Applied Biosystems). Samples were analysed on an ABI PRISM 310 Genetic Analyzer (PerkinElmer Applied Biosystems). Size of the alleles and area of the peaks were calculated with GeneScan 3.1 (Perkin-Elmer Applied Biosystems). Statistical analysis was performed with SPSS 4.0. In order to assess whether duplication had occurred at any given locus, a quantitative analysis was performed. DNA from the proband and one or more controls with the same genotype was amplified, samples were collected at several points, depending on the kinetics of amplification of each amplimer. The area of each allelic peak (a measure of the amount of amplified material) and the ratio $\left(\mathrm{R}_{\mathrm{A1} / \mathrm{A2}}\right)$ between the areas of the shorter (A1) and longer (A2) allele were calculated. Analysing samples at multiple points during amplification helped to minimise PCR artifacts and gave additional information about the kinetics of amplification at each locus. 
a

\section{6,XX,dup(2)(qter q33)}

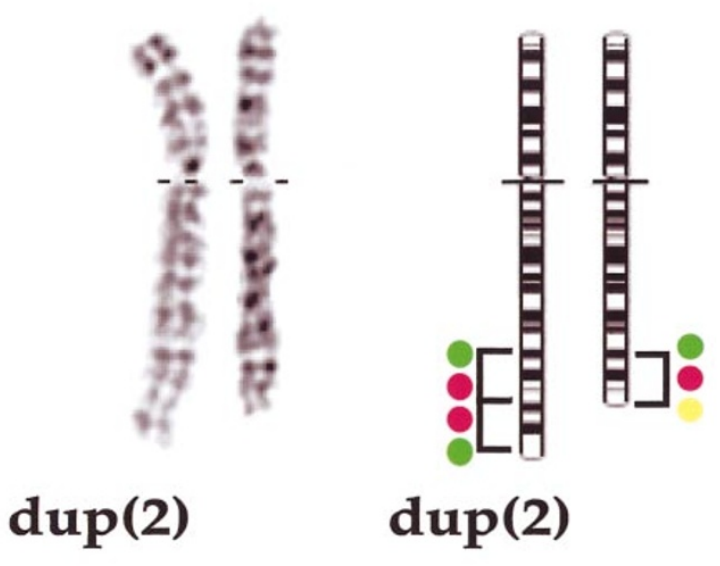

b

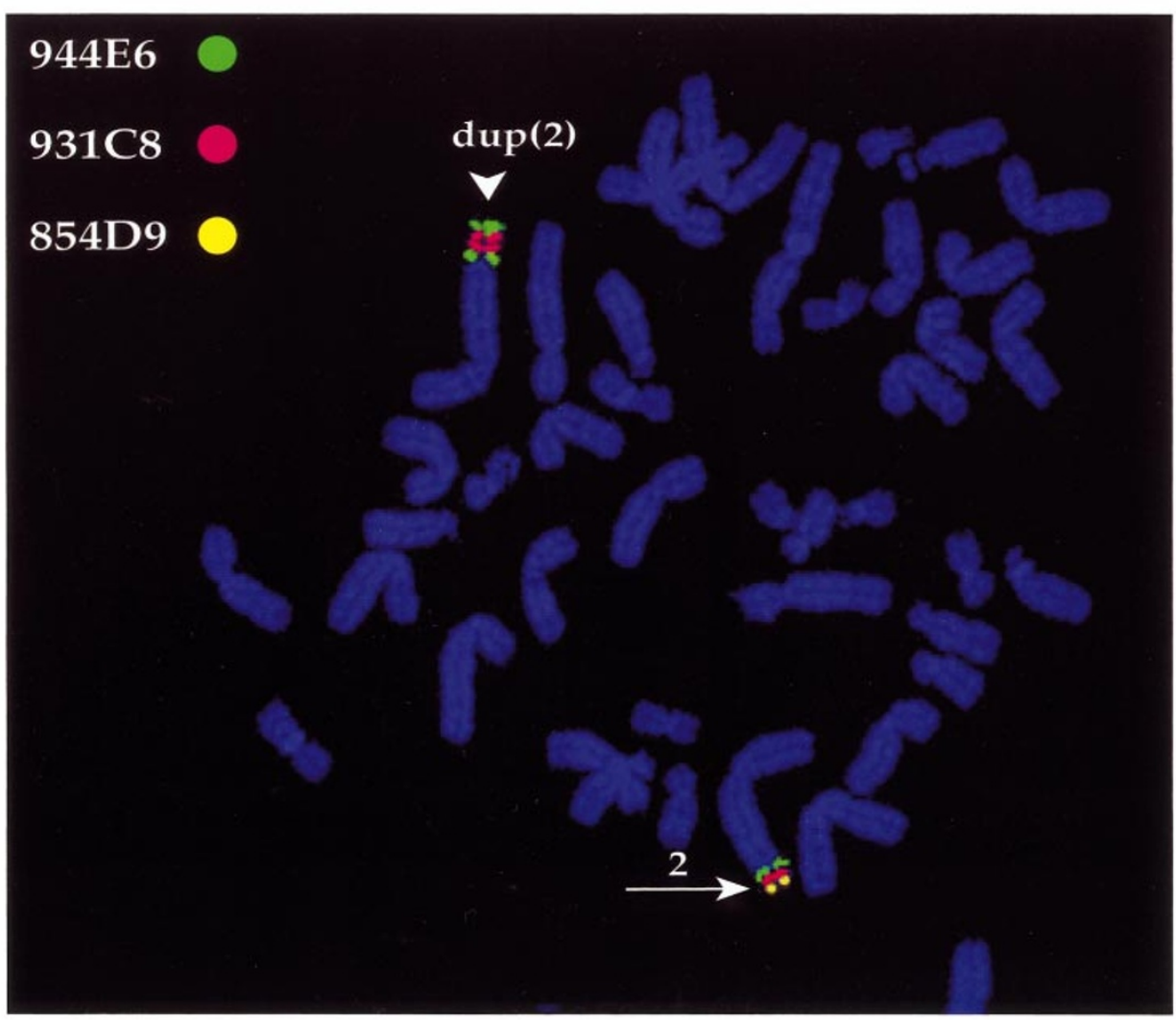

Figure 1 A (Left) cut-out of the abnormal and the normal chromosomes 2 in G-banding at a resolution of 550 bands; (right) ideogram of chromosomes 2 showing the deleted region (yellow dot) and the inverted duplicated one (green, red, red, green dots). B FISH with YACs spread along the distal 2q. The normal chromosome2 (arrow) has three signals, whereas the dup(2) (arrowhead) lacks the yellow signal. The order of red and green dots in the dup(2) demonstrates that the duplication is inverted. 


\section{Results}

Cytogenetic investigations consistently showed supernumeray bands on the distal long arm of one chromosome 2 (Figurela). Chromosome2 painting showed homogeneous staining along normal and abnormal chromosomes2. FISH with YACs from $2 q$ (Figure $1 b$ ) demonstrated a deletion of the last $10 \mathrm{cM}$ (from WI-6310 to the telomere) and a duplication of about $40 \mathrm{cM}$ (from D2S104 to WI-6310). Triple colour FISH (Figure 1b) revealed that the duplication was inverted. Cytogenetic investigations on her parents gave normal results. FISH and genotyping results of all informative loci tested are shown on Figure2. At loci D2S125 and D2S2253 neither maternal allele was transmitted to the proband, demonstrating the presence of a deletion of maternal origin (Figure3). A PCR-based assay for the presence of duplication was performed on all loci where the proband was heterozygous. For most amplimers $R_{A 1 / A 2}$ was quite consistent at all points analysed. $R_{A 1 / A 2}$ values were not significantly different in the proband and controls for the 2p loci D2S2216 and D2S2351, and the 2q loci D2S2248 and D2S301. At the remaining loci $R_{A 1 / A 2}$ values in proband and controls differed significantly. The results demonstrate that a duplication of maternal origin, extending at least from D2S104 to D2S2338 (Figures 2 and 3), occurred in the proband. We were unable to demonstrate transmission of both maternal alleles to the proband at any of the loci we tested, but we were able to exclude it for loci D2S2202, D2S2973, D2S407, D2S336, D2S331, D2S2297, D2S2158 and D2S104. It must be noted that FISH with YACs 805D2 and 890F11 gave only one signal, although the corresponding microsatellite analysis for loci D2S345, D2S338 indicated two copies on the rearranged chromosome (Figure2). This indicates that the two copies were too close together to be resolved by metaphase FISH.

\section{Discussion \\ Rearrangement}

Among inverted duplications, those better studied were invdup(8p)s of which about 30 cases have been reported. Floridia et $\mathrm{al}^{4}$ demonstrated that a distal deletion is always associated with the duplications and that, in spite of the variable size of the duplication, two regions have consistently the same size, that is the deleted region and the single copy region between the two duplicated regions. The finding that some of these cases are dicentric with a second inactive centromere at the tip of the invdup(8p) indicated that the formation of the new chromosome required two events: (1) the formation of a dicentric chromosome8qter-cen$8 p:: 8 p-c e n-8 q t e r$ and (2) its breakage at anaphase with the breakpoint at the level of the second centromere or more proximally along the short arm. The presence of a single copy region between the two regions in duplication indicates that the breakpoints at $8 p$ are not symmetric. In our invdup(2q), the distal region from D2S2253 to the telomere is deleted.

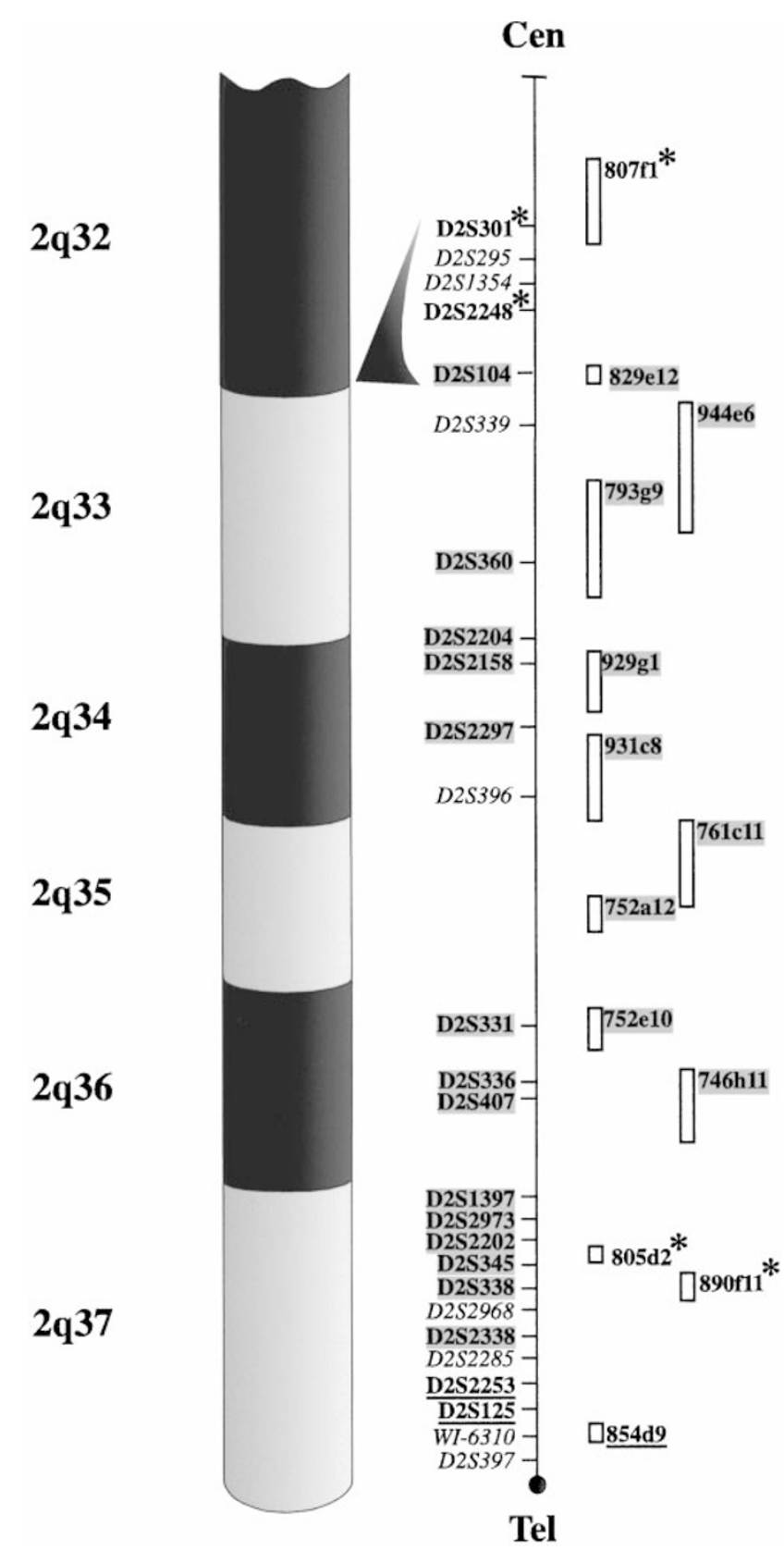

Figure 2 Ideogram of distal $2 q$ showing the portion of chromosome involved in the rearrangement. On the left of the line Cen-Tel are all the loci tested by microsatellite analysis. Loci in bold are informative; those in single copy in the invdup chromosome have an asterisk; the duplicated ones are on a grey ground; the deleted ones are underlined. On the right of the line, the bars indicate the YACs used for FISH: those with an asterisk give a single set of signals, those on a grey ground give two sets of signals; the underlined one gives signals only on the normal chromosome 2 .

The duplication region spans from D2S104 to D2S2338 (Figure2). 
D2S125

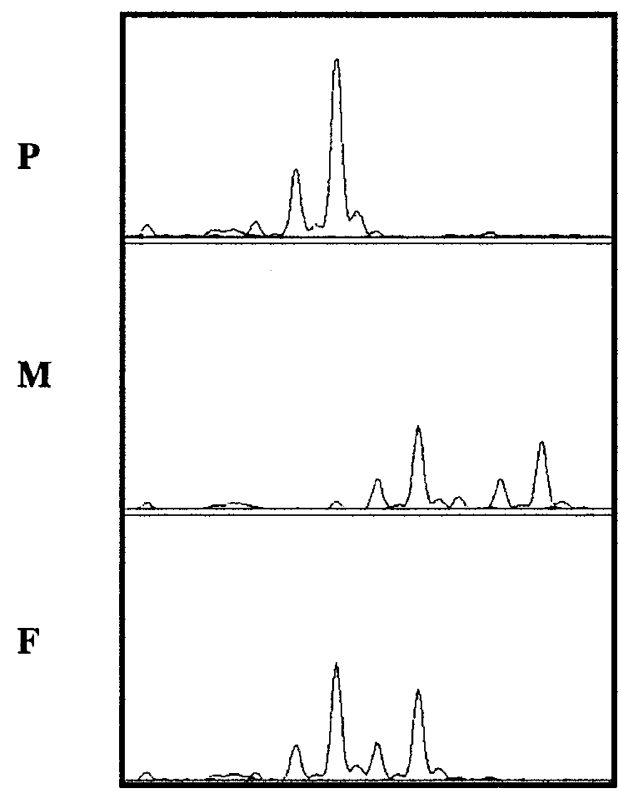

D2S2338

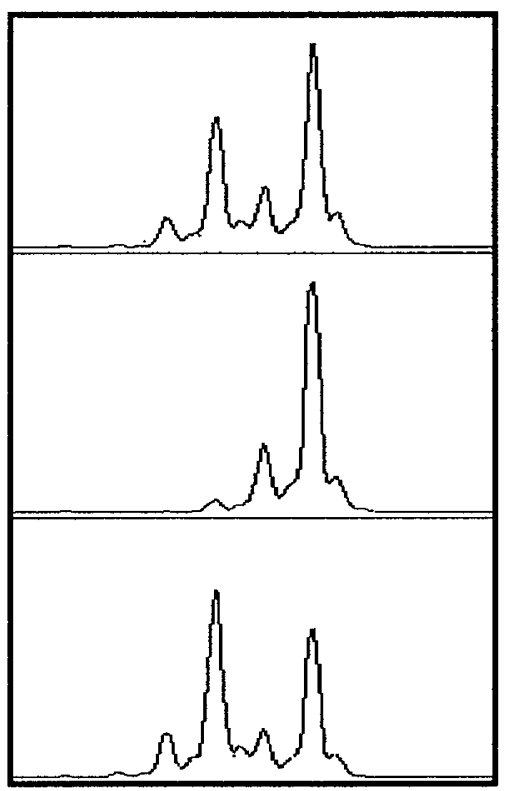

D2S104

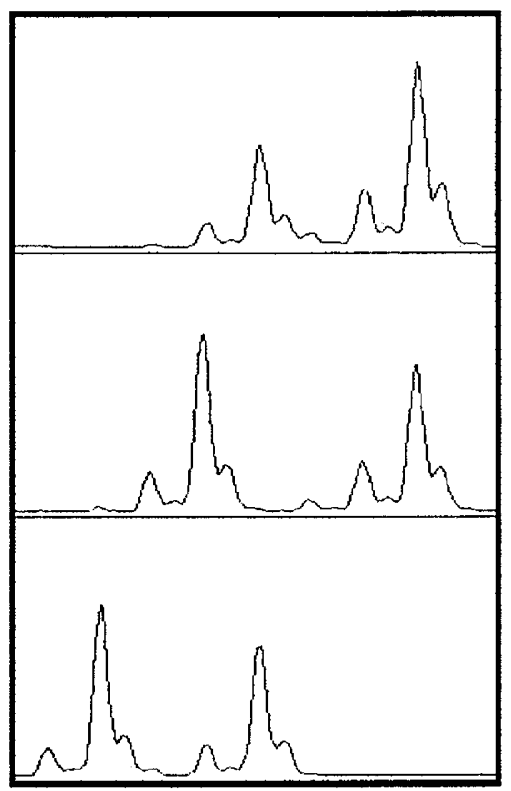

Figure 3 Microsatellite analysis of markers D2S125 (deleted), D2S2338 and D2S104 (duplicated) on ABI 310 genetic analyser. P: proband, M: mother; F: father. At the D2S125 locus the proband inherited neither of the mother's alleles. At loci D2S2338 and D2S104 the proband inherited a double dosage of one of the maternal alleles.

We hypothesised that, at meiosis I, the two homologues synapse for most of their length. The partial refolding of one homologue into itself (Figure4) would lead to the intrachromatid synapsis of the inverted repeats. In this way, after a single crossover, a recombinant chromosome, deleted for the region beyond the most distal repeat and with a single copy region located between the two repeats arises (Figure4). Indeed, the finding that in our invdup $(2 q)$, the entire $40 \mathrm{cM}$ duplication region involves only one of the two maternal alleles indicates that the abnormal crossover occurs between sister chromatids. At meiosis II, the two chromatids, linked together, can join the opposite poles provided that a breakage between the two centromeres occurs. If the breakage occurs asymmetrically, the two recombinant products are an inverted duplicated/deleted $2 q$, as in the case reported here, and a deleted $2 q$ with breakpoints from $2 q 35$ to $2 q 37$ as reported el sewhere. ${ }^{15,16}$ Since telomerase activity is expressed selectively in germ-line and malignant tumour cells but not in most normal human somatic cells, ${ }^{17}$ healing of the broken end must soon occur.

Although the mechanism we hypothesised predicts that the two duplicated regions should be separated by a single copy region, we were not able to demonstrate it. However, the single copy region could be localised between the two contigs WC-2.16 and WC-1572, a region that we did not analyse. Similarly to invdup(8p)s, which are invariably of maternal origin, ${ }^{4}$ our invdup( $\left.2 q\right)$ originated at the maternal meiosis as demonstrated by the absence of maternal alleles in the deleted region and the presence of a double dosage of maternal alleles in the duplication region (Figure3). However, in other cases of inverted duplications a paternal origin of the rearrangement has been demonstrated. ${ }^{9,18}$

According to the mechanism we proposed, the recurrence of the abnormal crossover in distal $2 q$ should be expected due to the presence of the inverted repeats. Two well known submicroscopic inversions caused by abnormal crossover between repeated inverted sequences disrupt the factor VIII and the IDS gene in distal $\mathrm{Xq}$ and occur in $45 \%$ of patients with severe haemophilia $A^{19}$ and in $13 \%$ of Hunter disease patients $\mathrm{s}^{20,21}$ respectively. At the macroscopic level, the recurrence of inverted duplication of $8 p$, invdup(8p), all with the same breakpoints, ${ }^{4}$ indicates that the events leading to this chromosomal rearrangement are not fortuitous and that the sequences at the breakpoints do predispose to abnormal recombination. The invdup( $2 q)$ seems also to be recurrent at least on cytogenetic grounds. The finding that no deletion has been reported in the three cases of $2 q$ inverted duplications $\mathrm{s}^{22-24}$ is probably due to the absence of molecular characterisation. In fact our case was interpreted at first as a distal $2 q$ duplication.

\section{Phenotype}

Our patient was clinically evaluated at birth owing to multiple congenital anomalies. At that time the cytogenetic analysis gave normal results and no diagnosis could be formulated. Distal $2 q$ deletions have been associated with an 
Figure 4 Overall mechanism of origin of the rearrangements resulting in inverted duplications concurrent with distal deletions. First meiotic division (MI): the two homologous chromosomes undergo canonic recombination along the synapsed portion. Repeated inverted sequences (dotted line and red arrows) localised in the same arm, favour the partial refolding of one chromosome allowing intrachromatid synapsis and ectopic recombination. Black arrows show how the recombinant chromosome (MI) originates. Black dots in the distal long arms indicate the region which will be deleted. At the second meiotic division (MII), when the centromere splits up the recombinant chromosome breaks asymmetrically (oblique line) originating an inverted duplicated/deleted chromosome (left part) and a deleted chromosome (right part). Note that in all the inverted duplicated/deleted chromosomes the duplication regions should be separated by a single copy region localised between the inverted repeats.

Albright hereditary osteodystrophy (AHO)-like syndrome (OMIM 600430) characterised by brachymetaphalangia, mental retardation and short stature. ${ }^{25}$ Wilson et $\mathrm{al}^{25}$ were able to narrow the $\mathrm{AHO}$-like critical region to $20 \mathrm{cM}$ in the distal 2q (from D2S338 to the telomere). Our patient does not have these characteristics, showing arach nodactyly of hands and feet and a stature around the 97th centile. Since her $2 q$ deletion spans the distal $10 \mathrm{cM}$ (from D2S2253 to the telomere), these findings halve the critical region for the skeletal malformations in AHO-like syndrome to $10 \mathrm{cM}$ from D2S338 to D2S125. The finding that among 33 subjects with 2q37 deletion, only 16 showed the AHO-like phenotype, ${ }^{25}$ indicates either that haploinsufficiency for the gene in the AHO-like critical region has reduced penetrance or that half of the $2 q 37$ deletions are interstitial. It is interesting to notice that both our patient and some of the cases with distal $2 q$ deletion ${ }^{26,27}$ display an aggressive behaviour with self-mutilation. Since our patient has the smallest $2 q$ deletion region, we assume that a dosage-sensitive gene affecting the behaviour is located in the distal $10 \mathrm{cM}$ of $2 \mathrm{q}$.

\section{Acknowledgements}

We are grateful to the YAC Screening Center of San Raffaele Biomedical Science Park (Milan, Italy) for providing the YACs and to the cell bank supported by Telethon (project C18). OZ and GS have been supported by cofin98-MURST (Ministero dell' Università e della Ricerca Scientifica e Tecnologica) and IRCCS Policlinico San Matteo, Pavia.

\section{References}

1 Taylor KM, Francke U, Brown MG, George DL, Kaufhold M: Inverted tandem ('mirror') duplications in human chromosomes: inv dup 8p, 4q, 22q. Am J Med Genet 1977; 1: 3-19.

2 Weleber RG, Verma RS, Kimberling WJ, Fieger HG Jr, Lubs HA: Duplication-deficiency of the short arm of chromosome8 following artificial insemination. Ann Genet 1976; 19: 241-247. 
3 Dill FJ, Schertzer M, Sandercock J, Tischler B, Wood S: Inverted tandem duplication generates a duplication deficiency of chromosome8p. Clin Genet 1987; 32: 109-113.

4 Floridia G, Piantanida M, Minelli et al: The same molecular mechanism at the maternal meiosis I produces mono and dicentric 8p duplications. Am J Hum Genet 1996; 58: 785-796.

5 Mewar R, Harrison W, Weaver DD, Palmer C, Davee MA, Overhauser J: Molecular cytogenetic determination of a deletion/ duplication of $1 q$ that results in a trisomy 18 syndromelike phenotype. Am J Med Genet 1994; 52: 178-183.

6 Jenderny J, Poetsch M, Hoeltzenbein M, Friedrich U, Jauch A: Detection of a concomitant distal deletion in an inverted duplication of chromosome3. Is there an overall mechanism for the origin of such duplications/deficiencies? Eur J Hum Genet 1998; 6: 439-444.

7 Sreekantaiah C, Kronn D, Marinescu RC, Goldin B, Overhauser J: Characterisation of a complex chromosomal rearrangement in a patient with a typical catlike cry and no other clinical findings of cri-du-chat syndrome. Am J Med Genet 1999; 86: 264-268.

8 Hoo J, Chao M, Szego K, Rauer M, Echiverri SC, Harris C: Four new cases of inverted terminal duplication: a modified hypothesis of mechanism of origin. Am J Med Genet 1995; 58: 299-304.

9 Stetten G, Charity LL, Kasch LM et al: A paternally derived inverted duplication of $7 q$ with evidence of a telomeric deletion. Am J Med Genet 1997; 68: 76-81.

10 Teebi AS, Gibson L, McGrath J, Meyn MS, Breg WR, Yang-Feng TL: Molecular and cytogenetic characterisation of 9p- abnormalities. Am J Med Genet 1993; 46: 288-292.

11 Pangalos C, Theophile D, Sinet PM et al: No significant effect of monosomy for distal 21q22.3 on the Down syndrome phenotype in 'mirror' duplications of chromosome21. Am J Hum Genet 1992; 51: $1240-1250$.

12 Milunsky J, Huang XL, Wyandt HE, Milunsky A: Schizophrenia susceptibility gene locus at Xp22.3. Clin Genet 1999; 55: 455-460.

13 Dutrillaux B, Viegas-Pequignot $E$ : High resolution $R$ - and G-banding on the same preparation. Hum Genet 1981; 57: 93-95.

14 Carrozzo R, Arrigo G, Rossi E et al: Multiple congenital anomalies, brain hypomyelination, and ocular albinism in a female with $\operatorname{dup}(X)$ (pter $\rightarrow$ q24::q21.32 $\rightarrow$ qter) and random $X$ inactivation. Am J Med Genet 1997; 72: 329-334.
15 Schinzel A: In: Baraitser M, Winter RM (eds). The Human Cytogenetics Database. Oxford University Press, Oxford: 1994.

16 Conrad B, Dewald G, Christensen E, Lopez M, Higgins J, Pierpont ME: Clinical phenotype associated with terminal 2q37 deletion. Clin Genet 1995; 48: 134-139.

17 Prescott JC, Blackburn EH: Telomerase: Dr Jekyll or Mr Hyde? Curr Opin Genet Dev 1999; 9: 368-373.

18 Smith A, Jauch A, Slater H, Robson L, Sandanam T: Syndromal: Obesity due to paternal duplication 6(q24.3-q27). Am J Med Genet 1999; 84: 125-131.

19 Lakich D, Kazazian HH Jr, Antonarakis SE, Gitschier J: Inversions disrupting the factor VIII gene are a common cause of severe haemophilia A. Nat Genet 1993; 5: 236-241.

20 Bondeson $\mathrm{ML}$, Dahl N, Malmgren $\mathrm{H}$ et al: Inversion of the IDS gene resulting from recombination with IDS-related sequences is a common cause of the Hunter syndrome. Hum Mol Genet 1995; 4: 615-621.

21 Timms KM, Bondeson ML, Ansari-Lari MA et al: Molecular and phenotypic variation in patients with severe Hunter syndrome. Hum Mol Genet 1997; 6: 479-486.

$22 \mathrm{Yu} \mathrm{CW}$, Chen $\mathrm{H}$ : De novo inverted tandem duplication of the long arm of chromosome2(q34 leads to q37). Birth D efects Orig Artic Ser 1982; 18: 311-320.

23 Dahoun-Hadorn S, Bretton-Chappuis B: De novo inversionduplication of 2q35-2qter without growth retardation. Ann Genet 1992; 35: 55-57.

24 Seidahmed MZ, Rooney DE, Salih MA et al: Case of partial trisomy 2q3 with clinical manifestations of Marshall-Smith syndrome. Am J Med Genet 1999; 85: 185-188.

25 Reddy KS, Flannery D, Farrer RJ: Microdeletions of chromosome sub-band 2q37.3 in two patients with abnormal situs viscerum. Am J Med Genet 1999; 84: 460-468.

26 Wilson LC, Leverton K, Oude Luttikhuis MEM et al: Brachydactyly and mental retardation: an Albright hereditary osteodystrophylike syndrome localized to 2q37. Am J Hum Genet 1995; 56: 400-407.

27 Bijlsma EK, Aalfs CM, Sluitjer Set al: Familial cryptic translocation between chromosomes 2qter and 8qter: further delineation of the Albright hereditary osteodystrophy-like phenotype. J Med Genet 1999; 36: 604-609. 